\title{
Variations dans la composition du fromage "Télémé " au cours de sa maturation
}

\author{
par \\ C. MANOLKIDIS, A. POLYCHRONIADOU \\ et E. ALICHANIDIS \\ Laboratoire de Technologie Laitière de l'Université de Thessaloniki
}

\section{INTRODUCTION}

Le fromage "Féta » ainsi que le "Télémé » qui est de composition similaire, sont les fromages produits en la plus grande quantité en Grèce $[2,16]$. Ils sont tous les deux des fromages blancs, à pâte molle, préparés avec du lait de brebis (le plus souvent mélangé de lait de chèvre pour l'amélioration de leurs propriétés physiques et organoleptiques) ; ils possèdent une saveur piquante caractéristique et sont de production saisonnière.

Ils sont préparés par coagulation du lait à $24-30^{\circ} \mathrm{C}$ pendant $1 \mathrm{~h}$ [34], le plus souvent avec addition préalable d'une culture de bactéries lactiques. La différence entre "Féta » et "Télémé » réside dans la manière de leur salage et de leur emballage. Le premier est salé en surface et emballé dans des barils de bois ; le second, au contraire, prend le premier sel dans la saumure et ensuite il est salé en surface et est emballé dans des récipients en fer blanc. En raison du salage abondant et de la mise rapide au réfrigérateur, quelques jours seulement après la préparation, l'acide lactique produit pendant les premières étapes de la préparation du "Télémé " ne peut pas être consumé, d'où sa plus grande acidité [34] en comparaison avec "Féta ».

En fait, tant pour "Féta " que pour " Télémé » il n'y a pas de délimitation entre la maturation et l'emmagasinage. La maturation continue pendant toute la durée du séjour des fromages dans les réfrigérateurs. Et, comme la vente des fromages dépend surtout de la demande, on constate souvent que l'on vend des fromages insuffisamment mûrs ; sans que cela puisse être décelé par le Service de la Répression des Fraudes.

Malgré la consommation étendue de ces fromages, ce n'est que dans ces cinq dernières années que l'on s'est efforcé d'une part de les standardiser (actuellement leur composition et surtout leur 
saveur dépend du producteur et de la saison de production) et de l'autre, de trouver un procédé de contrôle de leur degré de maturation [21].

Dans ce but nous avons commencé, il y a trois ans, l'étude de la maturation du fromage "Télémé »; ce travail est le premier d'une série qui comprend les résultats de cette étude.

\section{QUELQUES MOTS SUR LA MATURATION DU FROMAGE}

La maturation du fromage est un phénomène fort complexe qui transforme la masse indigeste et sans saveur ni odeur du caillé en fromage consommable et savoureux. Et nous pouvons dire que la maturation a déjà commencé pendant ia préparation du fromage, étant donné que cette première étape est bien décisive pour sa marche.

On sait maintenant que la maturation est surtout caractérisée par la dégradation des protéines. On a constaté que cette protéolyse est plus étendue que profonde [30]. Elle se poursuit en deux phases qui se succèdent ou bien qui ont lieu en même temps [28]. La première, plus simple, consiste dans la décomposition hydrolytique de la protéine en albumoses, peptones, peptides et, finalement, en acides aminés. La deuxième, beaucoup plus compliquée, comprend la décomposition des acides aminés en composés exempts d'azote (surtout en acides gras) et ammoniaque. Conséquence de cette hydrolyse de la protéine est l'accroissement des substances azotées hydrosolubles [29] qu'on remarque pendant la maturation et, ensuite, leur décroissance [23].

Les autres constituants du caillé ne sont pas moins convertibles. Ils se transforment en donnant naissance à des composants nouveaux produisant le goût et l'arôme caractéristiques de chaque sorte de fromage. Ces changements dépendent surtout de l'action des enzymes, des micro-organismes participant à la maturation et, secondairement, il s'agit de phénomènes purement chimiques.

Ce sont surtout les bactéries lactiques qui affectent la marche de la maturation, d'une part, par la décomposition du lactose et, d'autre part, par les ferments protéolytiques libérés de leurs corps après leur mort $[15,32]$. Les ferments de la présure semblent avoir moins d'importance pour la maturation [15, 32]. Ainsi, la substitution de la présure (dont le prix ne fait que monter) par des ferments bactériens $[6,14,27]$, de la pepsine animale [22], des ferments végétaux [19], etc., a plusieurs fois donné des produits de la même qualité, tout en accélérant la maturation.

Il y a d'ailleurs un grand nombre de facteurs pouvant changer la vitesse (et même la marche) de la maturation. Ainsi, on a essayé avec succès l'utilisation de cultures de micro-organismes pour 
obtenir une meilleure qualité [24] et une accélération de la maturation de certains types de fromage $[7,20]$. On a aussi étudié le rôle de la température dans la maturation [31] et proposé diverses températures comme optimales pour la maturation de divers fromages $[3,4,5,13,25]$. On a, en outre, trouvé que les dimensions du fromage et l'utilisation de lait frais ou pasteurisé exercent quelque influence sur la maturation, ainsi que le salage [15] à cause de la création d'un milieu favorisant le développement sélectif de certains micro-organismes.

\section{PROVENANCE DES ECHANTILLONS ET METHODES DE CONTROLE}

Les échantillons du fromage à étudier étaient pris d'une fromagerie de la région de Thessaloniki. La préparation était faite sous la surveillance du personnel de notre laboratoire selon la méthode en usage en fromagerie. Le lait utilisé pour la préparation du fromage était du lait de brebis additionné de lait de chèvre dans la proportion de 12 p. 100 environ pendant l'hiver et de 17 p. 100 environ pendant le printemps et l'été. Avant d'être caillé, le lait était pasteurisé et partiellement écrémé. La composition du lait avant et après la pasteurisation et l'écrémage est donné dans le tableau 1.

$\mathrm{Au}$ lait à cailler on ajoutait du chlorure de chaux $\left(\mathrm{CaOCl}^{2}\right)$ dans la proportion de $400 \mathrm{~g} / \mathrm{t}$ de lait, ainsi qu'une culture de bactéries lactiques (culture pure de Streptococcus lactis et de Str. cremoris). Le caillage avait lieu à $30^{\circ} \mathrm{C}$ en $1 \mathrm{~h}$. Après la division et l'égouttage

TABLEAU 1

\begin{tabular}{|c|c|c|c|c|}
\hline & \multicolumn{2}{|c|}{ Lait produit en hiver } & \multicolumn{2}{|c|}{$\begin{array}{l}\text { Lait produit au printemps } \\
\text { et en été }\end{array}$} \\
\hline & $\begin{array}{l}\text { A la } \\
\text { réception }\end{array}$ & $\begin{array}{l}\text { Après la } \\
\text { pasteuri- } \\
\text { sation et } \\
\text { l'écrémage }\end{array}$ & $\begin{array}{l}\text { A la } \\
\text { réception }\end{array}$ & $\begin{array}{c}\text { Après la } \\
\text { pasteuri- } \\
\text { sation et } \\
\text { l'écrémage }\end{array}$ \\
\hline $\begin{array}{l}\text { Matière sèche (d'après } \\
\text { Fleischmann) } \ldots \ldots \ldots\end{array}$ & 17,72 p. 100 & 15,75 p. 100 & 18,66 p. 100 & 16,13 p. 100 \\
\hline $\begin{array}{llllll}\text { Acidité } & \ldots & \ldots & \ldots & \ldots & \ldots\end{array}$ & $9,09^{\circ} \mathrm{SH}$ & $8,41^{\circ} \mathrm{SH}$ & $8,87^{\circ} \mathrm{SH}$ & $9,39^{\circ} \mathrm{SH}$ \\
\hline $\begin{array}{lllllll}\mathrm{pH} & \ldots & \ldots & \ldots & \ldots & \ldots & \ldots\end{array}$ & 6,32 & 6,27 & 6,58 & 6,68 \\
\hline $\begin{array}{lllll}\text { Protéines } & \ldots & \ldots & \ldots & \ldots\end{array}$ & 5,11 p. 100 & 5,12 p. 100 & 5,12 p. 100 & 5,13 p. 100 \\
\hline Matière grasse & 7,3 p. 100 & 5,9 p. 100 & 7,8 p. 100 & 5,6 p. 100 \\
\hline $\begin{array}{llllll}\text { Lactose } & \ldots & \ldots & \ldots & \ldots & \ldots\end{array}$ & 5,03 p. 100 & 5,11 p. 100 & 5,04 p. 100 & 5,19 p. 100 \\
\hline Ions chlore $\ldots \ldots \ldots$. & $114,6 \mathrm{mg} / 100 \mathrm{ml}$ & $118,0 \mathrm{mg} / 100 \mathrm{ml}$ & $92,9 \mathrm{mg} / 100 \mathrm{ml}$ & $97,9 \mathrm{mg} / 100 \mathrm{ml}$ \\
\hline
\end{tabular}


du caillé, les morceaux du fromage $(13 \times 13 \times 10 \mathrm{~cm})$ étaient mis dans de la saumure $16^{\circ}$ Bé pendant $12 \mathrm{~h}$ à $25^{\circ} \mathrm{C}$. Ensuite, ils étaient placés dans des récipients en fer blanc et saupoudrés de sel. Salage et renversement des morceaux du fromage dans les pots avaient lieu à 3 ou 4 reprises et dans des intervalles de $12 \mathrm{~h}$. Finalement les morceaux du fromage étaient placés dans les pots d'emballage qui étaient fermés hermétiquement et introduits au réfrigérateur. Parallèlement on avait préparé de petits récipients d'une contenance d'un $\mathrm{kg}$ qui étaient transportés à notre laboratoire ( $8 \mathrm{j}$ après la préparation en hiver et $6 \mathrm{j}$ environ au printemps et en été). Ils étaient conservés à $7^{\circ} \mathrm{C}$ afin d'étudier leur maturation.

Nous avons ainsi suivi la maturation de 7 échantillons de fromages, ainsi prélevés, par des analyses successives pendant 6 à 9 mois et pendant 3 années de suite. Quatre séries d'analyses correspondaient à des fromages caillés pendant la période d'hiver (janviermars), les 3 autres séries à des fromages caillés pendant le printemps et l'été (avril-juin). Chaque série comprenait 14-16 échantillons pris d'abord deux fois par jour, ensuite une fois, et finalement à des intervalles de temps de plus en plus grands à cause de la variation très faible des composants du fromage, fait constaté pendant l'étude d'une série d'échantillons préliminaire.

Dans chaque échantillon nous déterminions l'humidité, l'acidité, le $\mathrm{pH}$, le lactose, les protéines, la matière grasse et les ions chlore. Le reste de l'échantillon était desséché dans des boîtes de Pétri à une température de $20^{\circ} \mathrm{C}$ sous vide (pression 10 Torr) et il était pulvérisé. Ensuite à l'aide d'éther sulfurique dans un appareil Sohxlet on faisait l'extraction, jusqu'à élimination complète, de la matière grasse. Nous triturions de nouveau la poudre dégraissée ainsi produite et, après évaporation complète de l'éther, nous la gardions dans un vase hermétiquement clos à la température de $-10^{\circ} \mathrm{C}$. De cette manière, nous la maintenions inaltérable afin de pouvoir l'utiliser pour la détermination des fractions des composants azotés. Et il a été prouvé que la conservation était parfaite car des analyses faites après un an ont donné les mêmes résultats que celles faites sur la poudre fraîchement préparée.

La détermination de l'humidité était faite par chauffage du fromage avec du sable à $105^{\circ} \mathrm{C}$ jusqu'à obtention d'un poids constant. Pour la détermination de l'acidité nous avions suivi la méthode décrite par Scormüller et al. [29]. C'est selon ces Auteurs que nous avions aussi exécuté la préparation du fromage pour mesurer le $\mathrm{pH}$ avec un pH-mètre Beckman $\mathrm{H}-3$.

Le lactose était déterminé selon la méthode de la F.I.L. pour la détermination du lactose dans le lait [11] et les ions chlore selon la méthode de la F.I.L. [10] également. La détermination de l'azote total était faite selon la méthode classique de Kjeldahl (coefficient de transformation de l'azote en protéines : 6,37), tandis que celle de la matière grasse d'après la méthode de Gerber - van Gulik. 
La teneur en matière grasse, protéines, lactose, ions chlore, de même que l'acidité (exprimée en acide lactique) était calculée en pourcentage de la matière sèche du fromage, de façon que les résultats fussent comparables.

\section{RESULTATS ET DISCUSSION}

Les résultats des déterminations mentionnées ci-dessus sont représentés par les courbes des figures 1 - 7 .

L'humidité du fromage (fig. 1) a montré pendant les premiers jours (et principalement pendant le salage) un décroissement très rapide. Au contraire, la perte de l'eau était moindre après la fermeture des pots mais elle continuait avec une vitesse de plus en plus décroissante pendant toute la période de la maturation. Un petit

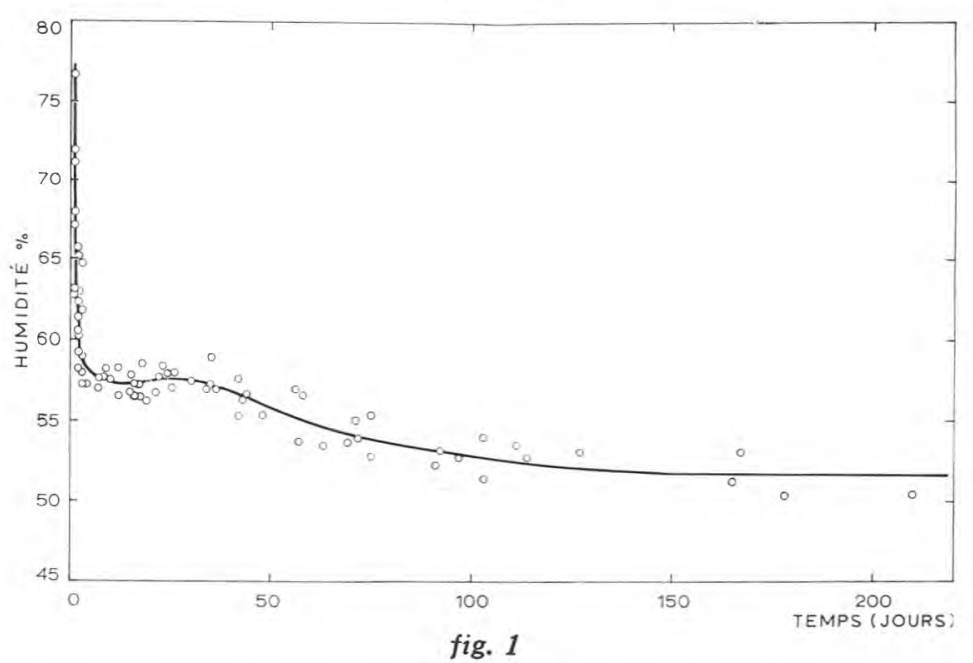

arrêt du décroissement est observé entre le $10^{\mathrm{me}}$ et le $30^{\mathrm{me}}$ jour (et même il a eu lieu une certaine augmentation de l'humidité entre le $22^{\text {me }}$ et le $28^{\text {me }}$ jour).

L'acidité (fig. 2) calculée comme pourcentage en acide lactique, a montré jusqu'au $100^{\mathrm{me}}$ jour un accroissement continu, d'abord rapide et ensuite plus lent. On a constaté une diminution analogue $\mathrm{du} \mathrm{pH}$ (fig. 3 ) jusqu'au $70^{\mathrm{me}}$ jour qui à la suite augmentait régulièrement jusqu'à la fin de la maturation.

Plusieurs auteurs $[17,30,29]$ ont remarqué un abaissement de l'acidité et une élévation du $\mathrm{pH}$ dès le commencement de la maturation. Au contraire, pendant la maturation du fromage "Télémé » on ne constate de décroissement de l'acidité que 100 jours après la coagulation et il se fait à une vitesse bien modérée. 




fig. 2

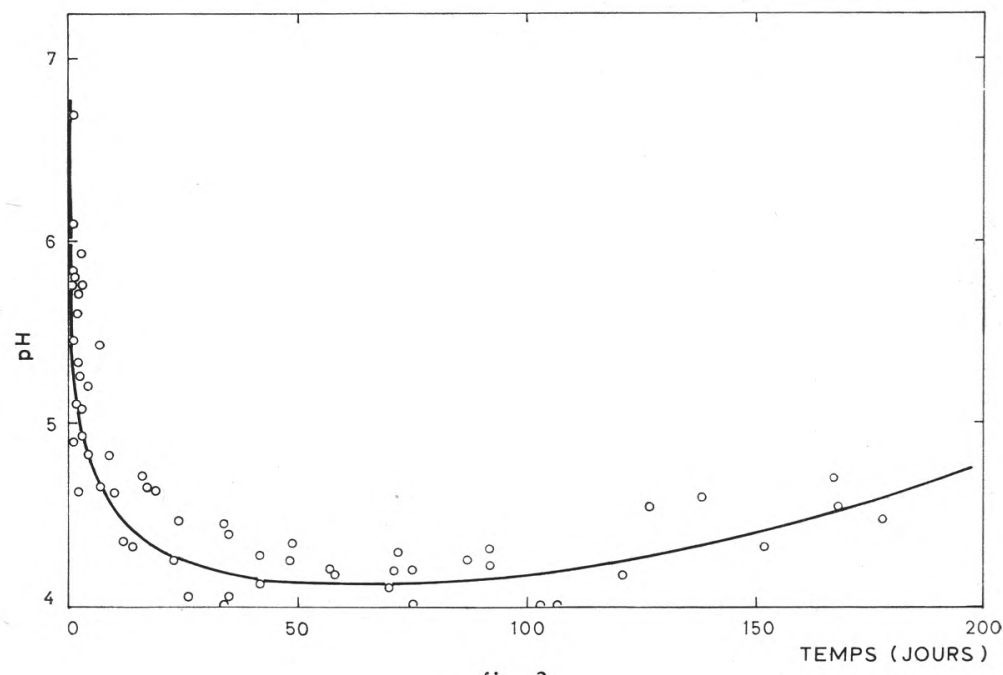

fig. 3



fig. 4 
En contradiction avec d'autres Auteurs [30, 21] nous n'avons pas constaté une disparition du lactose (et même dès les premiers jours). Après bien des fluctuations il s'est réduit presqu'à la moitié de sa concentration dans les 50 premiers jour (fig. 4), mais, ensuite, il a commencé a augmenter continuellement et progressivement il s'est rapproché après 6 mois du premier niveau. Cette augmentation pourrait être traduite comme résultant de la méthode oxydoréductrice que nous avons appliquée pour la détermination du lactose. Cela a eu pour conséquence la détermination simultanée d'autres substances réductrices et surtout du glucose et du galactose, produits d'hydrolyse du lactose. Ces monosaccharides sont dépensés de plus en plus lentement par les micro-organismes et sont accumulés en quantités considérables. Cet avis est corroboré par les résultats de Nakanishi et Tokita [23] qui constatent un accroissement du glucose dans le fromage en maturation et de Fagen et al. [8] qui trouvent pendant longtemps du galactose dans du fromage Cheddar en maturation.



fig. 5

L'azote total (fig. 5), calculé comme protéine, a montré un décroissement pendant les 10 premiers jours, évidemment à cause du passage dans le petit-lait (sérum) d'une grande partie des substances azotées solubles. Après la fermeture des pots et pendant 15 jours a eu lieu un accroissement temporaire de l'azote total suivi d'un graduel décroissement dû à la dégradation des protéines en substances azotées solubles (ayant passé dans le petit-lait) et ensuite en ammoniaque.

Le pourcentage en matière grasse (fig. 6) a montré généralement une augmentation normale bien que plus sensible pendant les 60 premiers jours. D'autres Auteurs [12, 33] également sont arrivés au même résultat, tandis que Sturm et Mair-Waldburg [30] expliquent cet accroissement par la détermination avec la matière grasse d'une partie des acides gras libérés pendant la maturation. Etant donné que la matière grasse est normalement hydrolysée pendant la maturation par les lipases des micro-organismes et du lait [1], l'accrois- 


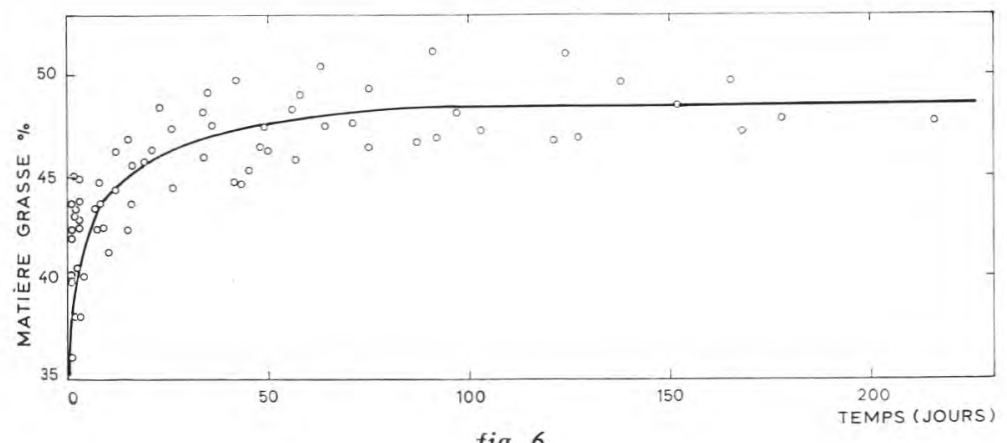

fig. 6

sement de la matière grasse par rapport à la matière sèche du fromage ne peut être justifié que comme résultant de la diminution des autres constituants, transformés en produits volatils (acides volatils, ammoniaque, anhydride carbonique).

Enfin la teneur en ions chlore (fig. 7) a considérablement augmenté pendant les 20 premiers jours à cause du salage fréquent et de l'éloignement en même temps du petit-lait. Nous avons essayé de prendre un échantillon avant chaque salage afin de connaître la quantité du sel absorbé par le salage précédent. Vers le $20^{\text {me }}$ jour après la coagulation a commencé une faible et irrégulière diminution du sel, due à la séparation d'une petite quantité de petit-lait pendant le séjour dans le réfrigérateur. Finalement, après le $150^{\text {me }}$ jour, le contenu en ions chlore a augmenté légèrement, fait ne pouvant être expliqué que par la diminution des autres constituants.



fig. 7

Généralement les grandes variations et changements dans les composants du fromage se sont presque limités aux 2 premiers mois suivant la coagulation. Après cela les changements des composants ont suivi une marche lente et plus normale. Mais les plus im- 
portants et plus intéressants changements ont été constatés sur les produits d'hydrolyse des protéines et, en général, aux fractions des substances azotées du fromage. Ces changements, pouvant aussi être utilisés comme un critère du degré de maturation du fromage, feront l'objet d'une prochaine publication.

Mais il est bien remarquable que, malgré le comportement uniforme des divers fromages examinés en ce qui concerne la marche chimique de leur maturation, ils n'avaient pas les mêmes caractères organoleptiques et n'étaient pas arrivés à la maturité dans le même temps. Etant donné que le traitement était le même, ce fait pourrait être expliqué, d'une part, par la composition différente du lait servant à leur production et, d'autre part, par le salage plus ou moins abondant. Ainsi, par exemple, les fromages produits pendant l'été, qui sont salés dans un temps plus court (pour empêcher la grande activité bactérienne due à la température élevée du milieu ambiant et à la provocation de fautes provenant d'elle) exigent un temps plus long pour obtenir la saveur et la texture désirées.

Ceci présente un intérêt spécial parce que plusieurs ont l'habitude de conserver le fromage "Télémé " dans la saumure. L'influence de cet excessif salage sur la marche de la maturation et les caractères organoleptiques du fromage vont faire l'objet d'une prochaine étude.

Reçu pour publication en juin 1969.

\section{Résumé}

Dans le présent travail nous avons étudié la maturation du fromage "Télémé " produit en hiver (janvier-mars) ou au printemps (avril-juin) dans la région de Thessaloniki et nous avons suivi la variation de ses principaux éléments.

Les changements les plus notables se sont montrés pendant les 60 premiers jours de la maturation (plus intenses pendant les 10 premiers jours), tandis que pendant les 7 mois suivants l'altération était lente et régulière.

La teneur en eau, protéines et chlorure de sodium (sel) a continuellement diminué, tandis que la matière grasse a montré un petit accroissement dû à la diminution des autres constituants de la matière sèche du fromage. Les hydrates de carbone réducteurs ont diminué pendant les premiers 27 - 45 jours tandis que, plus tard, ils ont montré un accroissement dû probablement à l'hydrolyse du lactose et à la décomposition incomplète des monosaccharides produits. Enfin, après un accroissement pendant 100 jours, l'acidité a commencé à décroître et, en même temps, le $\mathrm{pH}$ a pris des valeurs de plus en plus grandes.

Les propriétés organoleptiques des fromages n'ont pas suivi nettement les changements de la composition chimique et la matura- 
tion de chaque fromage a été accomplie dans des intervalles de temps bien différents.

\section{Su m m a ry}

The ripening of "Teleme » white cheese is examined. Samples of it have been prepared during winter (january-march) and spring (april-june) in Thessaloniki area.

The most important changes of its constituents have taken place in the first 60 days of the ripening process, particularly in the first 10 days. The changes during another 100 days were slow and of a regular character.

The water, protein and sodium chloride content was continually reduced while the fat content showed a small increase, due to the decrease of the dry material of the cheese. The reducing sugars appeared reduced in the first 27-45 days but they were increased later, probably because of hydrolysis of lactose and partial decomposition of the monosaccharides produced. The acidity kept increasing in the first 100 days but decreasing in the remaining time.

\section{Bibliographie}

[1] Alichanidis (E.S.) (1968), - La cinétique de l'action de la lipase du lait. Bull. annuel de la Faculté d'Agr. de l'Univ. de Thessaloniki, 81.

[2] Apostolou (A.) et Panajotopoulos (P.) (1967). - Die Milchwirtschaft Griechenlands. Milchwiss., 22, 496.

[3] Denkov (T.), Michailova (T.) et Marinova (E.) (1967). - Contribution to the temperature conditions for ripening of white brine cheese from sheep's Milk and determination of the moment when the cheese must be stored in refrigerated stores, Bulletin of Dairy res. Institut, Vidin, Bulg., 2, 25.

[4] Dimov (N.), Kozjev (A.) et Velev IS.) (1967). - Temperature conditions for kashkaval ripening. Bull. of Dairy res. Institut, Vidin Bulg., 2, 51.

[5] Dimov (N.), KozJev (A.) et Velev (S.) (1967). - Ripening temperature and stage of ripening for cold storage of Kachkaval cheese. Khranit. Prom., 16, 42.

[6] Emmanuiloff (I.) (1956). - Das Vorkommen freier Aminosäuren im mit bakteriellen Proteasen bwz. Labferment bereiteten bulgarischen weissen Schafmilchkaese. Int. Dairy Congr., 2, 200.

[7] ERIKSson (I.) et SJöström (G.). (1967). - Der Einfluss eines Wärmeschocks auf Säurewecker und dessen eventuelle Anwendung zur Beschleunigung der Käsereifung. Svenska mejeritidningen, 59, 219 et 235. Ref. Milchwiss., 22, 522 .

[8] Fagen (H. J.), Stine (J. B.) et Hussong (R. W.) (1952), - The identification of reducing sugars in Cheddar cheese during the early stages of ripening. J. Dairy Sci., 35, 779.

[9] F. I. L. Norme Internationale (1964), 26.

[10] F. I. L. Norme Internationale (1961), 17.

[11] F. I. L. Norme Internationale (1958), 4.

[12] Grigorov (H.) (1965) (1967). - Biochemische und mikrobiologische Veränderungen in der Zusammensetzung von Balkan-Kasche-Kaval während seiner Herstellung, Reifung und Lagerung. 2. Mitt. Biochemische Veränderungen von Balkan-Kasche-Kaval während der Reifung und Lagerung. Veter. Science (Sofia), 2, 745. Ref. in Milchwiss, 22, 186. 
[13] Hansen (E. C) (1946) (1950). - Influence de la température sur la maturation du fromage de Cheddar. Canad. Dairy Ice Cream J., 25 (6), 48. Ref. in Le lait, 30, 300.

[14] Higgs (L. T.) et Rogers (W. R.) (1967). - Microbial rennets. Austr. J. Dairy Technol., 22, 159.

[15] Inichov (G.S.) (1959), - Biochemie der Milch und der Milchprodukte. Vlg. Technik, Berlin.

[16] Iordanidis (I.) (1965), - La fromagerie en Grèce. Ed. du Ministère d'Agric., Athènes.

[17] KIKUCHI (T.) (1966). - Observations on Camembert cheese ripening. 17th Intern. Dairy Congr. D, 313.

[18] Koestler (G.) (1949). - Ein Beitrag zur Frage der Käsereifung. Milchwiss., 4, 269.

[19] Krishnamutri (C. R.) et Subrahmanyan (V.). Réf. Génin (G.) (1968). - Les succédanés de la présure. Le Lait, 48, 53.

[20] Menshikov (N.) (1966). - Acceleration of cheese ripening with increased proportions of lactic acid cultures and introduction of buffer salts. 17th Inter. Dairy Congr. D, 565.

[21] Etude sur le fromage grec (1963, 1964, 1965). - Ministère du Commerce, Athènes.

[22] Мотос (D.) et coll. Réf. GéniN (G.). (1968). — Les succédanés de la présure. Le Lait, 48, 53.

[23] Nakanishi (T.) et Tokita (F.) (1958), - Studies on Cheese ripening. Milchwiss, , 13, 9.

[24] Peters (I. I.) et Moore (A. V.) (1958), - Studies related to the manufacture of Swisstype cheese. II. Factors influencing ripening. J. Dairy Sci., 41, 74.

[25] RaAdeveld (C. W.) et MuldeR (H.) (1949). - Einfluss der Temperatur auf die Reifung des Edamer Käse. Neth. Milk Dairy J., 3, 117. Ref. Milchwiss., 4, 401.

[26] RaAdsveld (C. W.) (1953) (1956). - Composés amers du fromage. 13e Congr. Int. Lait., 2, 676. Réf. Le Lait, 36, 638.

[27] Robertson (P.S.) et coll. Réf. Génin (G.) (1968). — Les succédanés de la présure. Le Lait, 48, 53.

[28] Schormüller (J.) (1954). - Beiträge zur Biochemie der Käsereifung I. Mitt. Einleitung und Problemstellung. Zeitsch. Lebensmittel-Untersuch.Forsch., 98, 218.

[29] Schormüller (J.), Liese (H.) et Winter (H.) (1954). - Beiträge zur Biochemie der Käsereifung II. Mitt. Die Stickstoffbilanz im Verlauf der Reifung von Sauermilch - Magerkäse verschiedener Herstellungsart. Zeitsch. Lebensmittel-Untersuch.-Forsch., 98, 258.

[30] Sturm (W.) et MAIR-WALDBurg (H.) (1958). - Untersuchungen über Reifungsvorgänge bei Romadurkäse $30 \%$ Fett i. T. Ein Beitrag zur Chemie der Käsereifung. Milchwiss., 13, 167.

[31] Tноме́ (K. E.) (1949). - Der Einfluss niederer Lagertemperaturen auf die Reifung des Käses. 12th Int. Dairy Congr., 3, 217. Ref Milchwiss., 4, 401.

[32] Tofte Jespersen (N. I.) (1966). - Proteolytic activity of some lactic acid bacteria. 17th Int, Dairy Congr. D, 465.

[33] Wauschkuhn (B.). F.i. T. (1956), - Enderungen in Weichkäse während der der Reifung. Milchwiss, $11,176$.

[34] Zygouris (N.) (1952). - L'industrie laitière, 2e édition. Ministère de l'Agric., Athènes. 